\title{
Fixed points of multivalued nonself almost contractions in metric-like spaces
}

\author{
Hassen Aydi ${ }^{1} \cdot$ Abdelbasset Felhi $^{2} \cdot$ Slah Sahmim $^{2}$
}

Received: 19 March 2015 / Accepted: 23 May 2015/Published online: 17 June 2015

(c) The Author(s) 2015. This article is published with open access at Springerlink.com

\begin{abstract}
In this paper, we consider multivalued nonself weak contractions on convex metric-like spaces and we establish the existence of fixed point of such mappings. We provide some examples making effective our obtained result.
\end{abstract}

Keywords Hausdorff metric like - Almost contraction · Fixed point

Mathematics Subject Classification $47 \mathrm{H} 10 \cdot 54 \mathrm{H} 25$

\section{Introduction and preliminaries}

The study of fixed points for multivalued self mappings contractions using the Hausdorff metric was initiated by Nadler [16]. The fixed point theory for multivalued nonself mappings is developed by Assad and Kirk's [5]. They [5] proved the Banach's contraction principle for nonself multivalued mappings. For other results for multivalued nonself mappings, see $[3,9,10,14,17-19]$. On the other hand, Berinde [6, 7] introduced a new class of self mappings usually called weak contractions or almost contractions.

Hassen Aydi

hassen.aydi@isima.rnu.tn; hmaydi@uod.edu.sa

Abdelbasset Felhi

afelhi@kfu.edu.sa

Slah Sahmim

ssahmim@kfu.edu.sa

1 Department of Mathematics, College of Education of Jubail, University of Dammam, P.O. 12020, Industrial Jubail 31961, Saudi Arabia

2 College of Sciences, Department of Mathematics, KFU University, Al-hasa, Saudi Arabia
Recently, Alghamdi et al. [3] introduced the notion of multivalued nonself almost contractions as follows.

Definition 1.1 Let $(X, d)$ be a metric space and $K$ a nonempty subset of $X$. A map $T: K \rightarrow C B(X)$ is called a multivalued almost contraction if there exist a constant $k \in$ $(0,1)$ and some $L \geq 0$ such that

$$
H(T x, T y) \leq k d(x, y)+L d(y, T x) \text { for all } x, y \in K .
$$

Alghamdi et al. [3] proved the following fixed point theorem for multivalued nonself almost contractions on convex metric spaces.

Theorem 1.2 [3] Let $(X, d)$ be a complete convex metric space and $T: X \rightarrow C B(X)$ a multivalued almost contraction with $k \in(0,1)$ and some $L \geq 0$. If $k(1+L)<1$ and $T$ satisfies Rothe's type condition, that is, $x \in \partial K \Rightarrow T x \subset K$, then there exists $x \in K$ such that $x \in T x$.

In this paper, we extend the obtained results in [3] to the class of convex metric-like spaces. Mention that the concept of Hausdorff metric like was introduced in a very recent paper of Aydi et al. [4]. First, we need the following definitions and properties in the sequel.

Definition 1.3 Let $X$ be a nonempty set. A function $\sigma$ : $X \times X \rightarrow \mathbb{R}^{+}$is said to be a metric like (dislocated metric) on $X$ if for any $x, y, z \in X$, the following conditions hold:

$$
\begin{aligned}
& \left(\sigma_{1}\right) \sigma(x, y)=0 \Longrightarrow x=y ; \\
& \left(\sigma_{2}\right) \sigma(x, y)=\sigma(y, x) ; \\
& \left(\sigma_{3}\right) \sigma(x, z) \leq \sigma(x, y)+\sigma(y, z) .
\end{aligned}
$$

The pair $(X, \sigma)$ is then called a metric-like (dislocated metric) space.

Each metric-like $\sigma$ on $X$ generates a $T_{0}$ topology $\tau_{\sigma}$ on $X$ which has as a base the family open $\sigma$-balls $\left\{B_{\sigma}(x, \varepsilon)\right.$ : 
$x \in X, \varepsilon>0\}, \quad$ where $\quad B_{\sigma}(x, \varepsilon)=\{y \in X: \mid \sigma(x, y)-$ $\sigma(x, x) \mid<\varepsilon\}$, for all $x \in X$ and $\varepsilon>0$.

Observe that a sequence $\left\{x_{n}\right\}$ in a metric-like space $(X, \sigma)$ converges to a point $x \in X$, with respect to $\tau_{\sigma}$, if and only if $\sigma(x, x)=\lim _{n \rightarrow \infty} \sigma\left(x, x_{n}\right)$.

Definition 1.4 Let $(X, \sigma)$ be a metric-like space.

(a) A sequence $\left\{x_{n}\right\}$ in $X$ is said to be a Cauchy sequence if $\lim _{n, m \rightarrow \infty} \sigma\left(x_{n}, x_{m}\right)$ exists and is finite.

(b) $(X, \sigma)$ is said to be complete if every Cauchy sequence $\left\{x_{n}\right\}$ in $X$ converges with respect to $\tau_{\sigma}$ to a point $x \in X \quad$ such that $\lim _{n \rightarrow \infty} \sigma\left(x, x_{n}\right)=\sigma(x, x)=\lim _{n, m \rightarrow \infty} \sigma\left(x_{n}, x_{m}\right)$.

Every metric space is a metric-like space, but the converse may not be true.

Example 1.5 Let $X=\mathbb{R}$ and $\sigma: X \times X \rightarrow \mathbb{R}^{+}$defined by $\sigma(x, y)=|x|+|y| \quad$ for all $x, y \in X$.

Note that $\sigma$ is a metric like, but not a metric since $\sigma(1,1)=2>0$.

We need in the sequel the following trivial inequality

$\sigma(x, x) \leq 2 \sigma(x, y)$ for all $x, y \in X$.

For fixed point results for single-valued mappings in the setting of metric-like spaces, we may cite $[1,2,11,13,15$, 20, 21].

Very recently, Aydi et al. [4] introduced the concept of Hausdorff metric like. For instance, let $C B^{\sigma}(X)$ be the family of all nonempty, closed and bounded subsets of the metric-like space $(X, \sigma)$, induced by the metric-like $\sigma$. Note that the boundedness is given as follows: $A$ is a bounded subset in $(X, \sigma)$ if there exist $x_{0} \in X$ and $M \geq 0$ such that for all $a \in A$, we have $a \in B_{\sigma}\left(x_{0}, M\right)$, that is,

$\left|\sigma\left(x_{0}, a\right)-\sigma(a, a)\right|<M$.

The Closedness is taken in $\left(X, \tau_{\sigma}\right)$ (where $\tau_{\sigma}$ is the topology induced by $\sigma$ ). Let $\bar{A}$ be the closure of $A$ with respect to the metric-like $\sigma$. Then, if $A \in C B^{\sigma}(X)$, then $\bar{A}=A$. For $A \subset X$ and $a \in X$, we also have

$a \in \bar{A} \Longleftrightarrow B_{\sigma}(a, \varepsilon) \cap A \neq \emptyset \quad$ for all $\varepsilon>0$

For $A, B \in C B^{\sigma}(X)$ and $x \in X$, define

$$
\begin{aligned}
\sigma(x, A) & =\inf \{\sigma(x, a): a \in A\}, \delta_{\sigma}(\mathrm{A}, \mathrm{B}) \\
& =\sup \{\sigma(a, B): a \in A\}, \\
\delta_{\sigma}(B, A) & =\sup \{\sigma(b, A): b \in B\} \text { and } \mathrm{H}_{\sigma}(\mathrm{A}, \mathrm{B}) \\
& =\max \left\{\delta_{\sigma}(A, B), \delta_{\sigma}(B, A)\right\} .
\end{aligned}
$$

We find more details on the properties of $H_{\sigma}$ in [4]. We also have the following useful lemmas.

Lemma 1.6 Let $A, B \in C B^{\sigma}(X)$ and $a \in A$. Then, for all $\varepsilon>0$, there exists $a$ point $b \in B$ such that $\sigma(a, b) \leq H_{\sigma}(A, B)+\varepsilon$.

Lemma 1.7 [4] Let $(X, \sigma)$ be a metric-like space and $A$ be any nonempty set in $(X, \sigma)$, then

if $\sigma(a, A)=0, \quad$ then $a \in \bar{A}$.

We give the following definition concerning the concept of convexity on metric-like spaces. One may find its analog for the metric case in [5].

Definition 1.8 A metric-like space $(X, \sigma)$ is convex if for each $x, y \in X$ with $x \neq y$ there exists $z \in X, x \neq z \neq y$, such that

$\sigma(x, y)=\sigma(x, z)+\sigma(z, y)$.

We also need the following concepts.

Definition 1.9 Let $(X, \sigma)$ be a metric-like space and $A$ be a set in $X$. We have

$x \in \AA \Longleftrightarrow \exists \varepsilon>0, \quad B(x, \varepsilon) \subset A$,

where $B(x, \varepsilon)=\{y \in X,|\sigma(x, y)-\sigma(x, x)|<\varepsilon\}$. We define the boundary of $A$ in $(X, \sigma)$ as

ว $A=\bar{A} \backslash \AA$.

The purpose of this paper is to prove a fixed point theorem for multivalued nonself almost contractions on convex metric-like spaces. We derive many interesting corollaries on existing known results in the literature. Some examples are also presented illustrating our obtained result.

\section{Fixed point of multivalued almost contraction}

Now, we state and prove our main result.

Theorem 2.1 Let $(X, \sigma)$ be a complete metric-like space and $K$ a nonempty closed subset of $X$ such that if $x \in K$ and $y \notin K$, then there exists a point $z \in \partial K$ (the boundary of $K$ ) such that

$\sigma(x, y)=\sigma(x, z)+\sigma(z, y)$.

Suppose that $T: K \rightarrow C B^{\sigma}(X)$ is a multivalued almost contraction, that is,

$H_{\sigma}(T x, T y) \leq k \sigma(x, y)+L \sigma(y, T x)$, for all $x, y \in K$

with $k \in(0,1)$ and some $L \geq 0$ such that $(1+L)(k+$ $2 L)<1$. If $T$ satisfies Rothe's type condition, that is, $x \in$ 
$\partial K \Rightarrow T x \subset K$, then there exists $x^{\star} \in K$ such that $x^{\star} \in$ $T x^{\star}$, that is, $T$ has a fixed point in $K$.

Proof We construct a sequence $\left\{x_{n}\right\} \subset K$ in the following way:

Let $x_{0} \in K$ and $y_{1} \in T x_{0}$. If $y_{1} \in K$, let $x_{1}=y_{1}$. If $y_{1} \notin$ $K$, by (2.1) there exists $x_{1} \in \partial K$ such that

$$
\sigma\left(x_{0}, x_{1}\right)+\sigma\left(x_{1}, y_{1}\right)=\sigma\left(x_{0}, y_{1}\right) \text {. }
$$

We have $x_{1} \in \partial K$ and so by Definition 1.9, $x_{1} \in K$. Thus, by Lemma 1.6 , there exists $y_{2} \in T x_{1}$, such that

$$
\sigma\left(y_{1}, y_{2}\right) \leq H_{\sigma}\left(T x_{0}, T x_{1}\right)+k
$$

If $y_{2} \in K$, let $x_{2}=y_{2}$. If $y_{2} \notin K$, by (2.1) there exists $x_{2} \in$ $\partial K$ such that

$$
\sigma\left(x_{1}, x_{2}\right)+\sigma\left(x_{2}, y_{2}\right)=\sigma\left(x_{1}, y_{2}\right) \text {. }
$$

Therefore, $x_{2} \in K$. From Lemma 1.6, there exists $y_{3} \in T x_{2}$, such that

$$
\sigma\left(y_{2}, y_{3}\right) \leq H_{\sigma}\left(T x_{1}, T x_{2}\right)+k^{2} \text {. }
$$

Continuing in this fashion, we construct two sequences $\left\{x_{n}\right\}$ and $\left\{y_{n}\right\}$ such that

(i) $y_{n+1} \in T x_{n}$;

(ii) $\sigma\left(y_{n}, y_{n+1}\right) \leq H_{\sigma}\left(T x_{n-1}, T x_{n}\right)+k^{n}$, where

(iii) $x_{n}=y_{n}$ if $y_{n} \in K$;

(iv) $\quad x_{n} \neq y_{n}$ if $y_{n} \notin K$ and then $x_{n} \in \partial K$ such that

$$
\sigma\left(x_{n-1}, x_{n}\right)+\sigma\left(x_{n}, y_{n}\right)=\sigma\left(x_{n-1}, y_{n}\right) \text {. }
$$

Mention that in the case (iv), $x_{n} \in \partial K$ and by Rothe's type condition, $y_{n+1} \in T x_{n} \in K$. Let

$$
\begin{aligned}
& P_{1}=\left\{x_{i} \in\left\{x_{n}\right\}: x_{i}=y_{i}, i=1,2, \ldots\right\} \quad \text { and } \\
& P_{2}=\left\{x_{i} \in\left\{x_{n}\right\}: x_{i} \neq y_{i}, i=1,2, \ldots\right\} .
\end{aligned}
$$

Note that, if $x_{n} \in P_{2}$ for some $n$, then $x_{n+1}, x_{n-1} \in P_{1}$. Now, for $n \geq 2$, three cases should be considered.

Case $1 x_{n}, x_{n+1} \in P_{1}$. Then, $y_{n}=x_{n}$ and $y_{n+1}=x_{n+1}$. Thus, using (1.2)

$$
\begin{aligned}
\sigma\left(x_{n}, x_{n+1}\right) & =\sigma\left(y_{n}, y_{n+1}\right) \\
& \leq H_{\sigma}\left(T x_{n-1}, T x_{n}\right)+k^{n} \\
& \leq k \sigma\left(x_{n-1}, x_{n}\right)+L \sigma\left(x_{n}, T x_{n-1}\right)+k^{n} \\
& \leq k \sigma\left(x_{n-1}, x_{n}\right)+L \sigma\left(x_{n}, x_{n}\right)+k^{n} \\
& \leq(k+2 L) \sigma\left(x_{n-1}, x_{n}\right)+k^{n} .
\end{aligned}
$$

Case $2 x_{n} \in P_{1}$ and $x_{n+1} \in P_{2}$. Then, $y_{n}=x_{n}$ and $y_{n+1} \neq$ $x_{n+1}$. In this case, we have by (iv),

$$
\begin{aligned}
\sigma\left(x_{n}, x_{n+1}\right) & \leq \sigma\left(x_{n}, x_{n+1}\right)+\sigma\left(x_{n+1}, y_{n+1}\right) \\
& =\sigma\left(x_{n}, y_{n+1}\right)=\sigma\left(y_{n}, y_{n+1}\right) \\
& \leq H_{\sigma}\left(T x_{n-1}, T x_{n}\right)+k^{n} \\
& \leq k \sigma\left(x_{n-1}, x_{n}\right)+L \sigma\left(x_{n}, T x_{n-1}\right)+k^{n} \\
& \leq(k+2 L) \sigma\left(x_{n-1}, x_{n}\right)+k^{n} .
\end{aligned}
$$

Case $3 x_{n} \in P_{2}$ and $x_{n+1} \in P_{1}$. Then, $x_{n} \neq y_{n}, x_{n-1}=y_{n-1}$, $x_{n+1}=y_{n+1}$ and $y_{n} \in T x_{n-1}$. We have

$$
\begin{aligned}
\sigma\left(x_{n}, x_{n+1}\right) & \leq \sigma\left(x_{n}, y_{n}\right)+\sigma\left(y_{n}, x_{n+1}\right) \\
& =\sigma\left(x_{n}, y_{n}\right)+\sigma\left(y_{n}, y_{n+1}\right) \\
& \leq \sigma\left(x_{n}, y_{n}\right)+k \sigma\left(x_{n-1}, x_{n}\right)+L \sigma\left(x_{n}, T x_{n-1}\right)+k^{n} .
\end{aligned}
$$

Since $k<1$ and $y_{n} \in T x_{n-1}$, then

$$
\begin{aligned}
\sigma\left(x_{n}, x_{n+1}\right) & <\sigma\left(x_{n}, y_{n}\right)+\sigma\left(x_{n-1}, x_{n}\right)+L \sigma\left(x_{n}, y_{n}\right)+k^{n} \\
& \leq \sigma\left(x_{n-1}, y_{n}\right)+L \sigma\left(x_{n}, y_{n}\right)+k^{n} \\
& =\sigma\left(x_{n-1}, y_{n}\right)+L\left[\sigma\left(x_{n-1}, y_{n}\right)-\sigma\left(x_{n-1}, x_{n}\right)\right]+k^{n} \\
& \leq(1+L) \sigma\left(y_{n-1}, y_{n}\right)+k^{n} \\
& \leq(1+L)\left[H_{\sigma}\left(T x_{n-2}, T x_{n-1}\right)+k^{n-1}\right]+k^{n} \\
& \leq(1+L)\left[k \sigma\left(x_{n-2}, x_{n-1}\right)+L \sigma\left(x_{n-1}, T x_{n-2}\right)\right] \\
& +(1+L) k^{n-1}+k^{n} \\
& \leq(1+L) k \sigma\left(x_{n-2}, x_{n-1}\right)+(1+L) L \sigma\left(x_{n-1}, x_{n-1}\right) \\
& +(1+L) k^{n-1}+k^{n} \\
& \leq(1+L)(k+2 L) \sigma\left(x_{n-2}, x_{n-1}\right)+(1+L) k^{n-1}+k^{n} .
\end{aligned}
$$

Since $h:=(1+L)(k+2 L)<1$, then

$$
\sigma\left(x_{n}, x_{n+1}\right) \leq h \sigma\left(x_{n-2}, x_{n-1}\right)+h k^{n-2}+k^{n} \text {. }
$$

Mention that $0<k \leq h<1$. Thus, due to above three cases, we deduce for $n \geq 2$

$$
\sigma\left(x_{n}, x_{n+1}\right) \leq\left\{\begin{array}{c}
h \sigma\left(x_{n-1}, x_{n}\right)+h^{n}, \text { or } \\
h \sigma\left(x_{n-2}, x_{n-1}\right)+h^{n-1}+h^{n} .
\end{array}\right.
$$

Let

$\alpha=\max \left\{\sigma\left(x_{0}, x_{1}\right), \sigma\left(x_{1}, x_{2}\right)\right\}$.

Following [5], by induction it follows that for $n \geq 1$,

$$
\sigma\left(x_{n}, x_{n+1}\right) \leq h^{\frac{n-1}{2}} \alpha+h^{\frac{n}{2}} n \text {. }
$$

Now, for $n>m$, we have

$$
\begin{aligned}
\sigma\left(x_{n}, x_{m}\right) & \leq \sum_{i=m}^{n-1} \sigma\left(x_{i}, x_{i+1}\right) \leq \alpha \sum_{i=m}^{n-1} h^{\frac{i-1}{2}}+\sum_{i=m}^{n-1} i h^{\frac{i}{2}} \\
& \leq \alpha \sum_{i=m}^{\infty} h^{\frac{i-1}{2}}+\sum_{i=m}^{\infty} i h^{\frac{i}{2}} \rightarrow 0 \text { as } m \rightarrow \infty .
\end{aligned}
$$


Since $\sum_{n=1}^{\infty} h^{n}$ converges, so

$\lim _{n, m \rightarrow \infty} \sigma\left(x_{n}, x_{m}\right)=0$.

Hence, $\left\{x_{n}\right\}$ is Cauchy in $(K, \sigma)$. Since $K$ is closed and $(X, \sigma)$ is complete, then $(K, \sigma)$ is complete. Thus, $\left\{x_{n}\right\}$ converges to a point $x^{\star} \in K$, that is,

$\lim _{n \rightarrow \infty} \sigma\left(x_{n}, x^{\star}\right)=\sigma\left(x^{\star}, x^{\star}\right)=\lim _{n, m \rightarrow \infty} \sigma\left(x_{n}, x_{m}\right)=0$.

We will show that $x^{\star}$ is a fixed point of $T$.

Observe that, by construction of $\left\{x_{n}\right\}$, there exists a subsequence $\left\{x_{n(p)}\right\}$ of $\left\{x_{n}\right\}$ each of whose terms is in the set $P_{1}$, (i.e, $\left.x_{n(p)}=y_{n(p)}, p=1,2, \ldots\right)$. Thus, by (i), $x_{n(p)}=y_{n(p)} \in T x_{n(p)-1}$.

We have, for all $p=1,2, \ldots$

$$
\begin{aligned}
\sigma\left(x^{\star}, T x^{\star}\right) & \leq \sigma\left(x^{\star}, x_{n(p)+1}\right)+\sigma\left(x_{n(p)+1}, T x^{\star}\right) \\
& \leq \sigma\left(x^{\star}, x_{n(p)+1}\right)+H_{\sigma}\left(T x_{n(p)}, T x^{\star}\right) \\
& \leq \sigma\left(x^{\star}, x_{n(p)+1}\right)+k \sigma\left(x_{n(p)}, x^{\star}\right)+L \sigma\left(x^{\star}, T x_{n(p)}\right) .
\end{aligned}
$$

Since,

$\lim _{p \rightarrow \infty} \sigma\left(x^{\star}, x_{n(p)+1}\right)=\lim _{p \rightarrow \infty} \sigma\left(x_{n(p)}, x^{\star}\right)=\lim _{p \rightarrow \infty} \sigma\left(x^{\star}, T x_{n(p)}\right)=0$,

then

$\sigma\left(x^{\star}, T x^{\star}\right)=0$.

Hence, by Lemma 1.7, $x^{\star} \in \overline{T x^{\star}}=T x^{\star}$. Then, $x^{\star}$ is a fixed point of $T$.

We state the following simple corollaries as consequences of Theorem 2.1.

Corollary 2.2 Let $(X, \sigma)$ be a complete metric-like space and $K$ a nonempty closed subset of $X$ such that: if $x \in K$ and $y \notin K$, then there exists a point $z \in \partial K$, (the boundary of $K$ ) such that

$\sigma(x, y)=\sigma(x, z)+\sigma(z, y)$.

Suppose that $T: K \rightarrow C B^{\sigma}(X)$ is a multivalued contraction, that is,

$H_{\sigma}(T x, T y) \leq k \sigma(x, y), \quad$ for all $x, y \in X$

with $k \in(0,1)$. If $T$ satisfies Rothe's type condition, that is, $x \in \partial K \Rightarrow T x \subset K$, then there exists $x^{\star} \in K$ such that $x^{\star} \in T x^{\star}$, that is, Thas a fixed point in $K$.

Proof It suffices to take $L=0$ in Theorem 2.1.

The metric case of Corollary 2.2 is
Corollary 2.3 [5] Let $(X, \sigma)$ be a complete convex metric space and $K$ a nonempty closed subset of $X$. Suppose that $T: K \rightarrow C B^{\sigma}(X)$ is a multivalued contraction, that is,

$H_{\sigma}(T x, T y) \leq k \sigma(x, y), \quad$ for all $\quad x, y \in X$

with $k \in(0,1)$. If $T$ satisfies Rothe's type condition, that is, $x \in \partial K \Rightarrow T x \subset K$, then there exists $x^{\star} \in K$ such that $x^{\star} \in T x^{\star}$, that is, $T$ has a fixed point in $K$.

We give the following illustrated examples.

Example 2.4 Let $X=[0, \infty), K=[0,1]$ and $k=\frac{1}{6}$. Consider $T: K \rightarrow C B^{\sigma}(X)$ given by $T x=\left\{0, \frac{x+5}{6}\right\}$, for all $x \in K$. Take $\sigma(x, y)=|x-y|$.

Mention that Rothe's type condition is easily verified. We show that $T$ is a multivalued almost contraction. In fact, we have for all $x, y \in[0,1]$

$$
\begin{aligned}
H_{\sigma}(T x, T y)= & \max \left\{\delta_{\sigma}(T x, T y), \delta_{\sigma}(T y, T x)\right\} \\
= & \max \left\{\max \left\{\sigma(0, T y), \sigma\left(\frac{x+5}{6}, T y\right)\right\},\right. \\
& \left.\max \left\{\sigma(0, T x), \sigma\left(\frac{y+5}{6}, T x\right)\right\}\right\} .
\end{aligned}
$$

Recall that

$$
\begin{aligned}
\sigma(0, T y) & =\min \{\sigma(0, b): b \in T y\} \\
& =\min \left\{\sigma(0,0), \sigma\left(0, \frac{y+5}{6}\right)\right\}=0,
\end{aligned}
$$

and

$$
\begin{aligned}
\sigma\left(\frac{x+5}{6}, T y\right) & =\min \left\{\sigma\left(\frac{x+5}{6}, b\right) ; b \in T y\right\} \\
& =\min \left\{\sigma\left(\frac{x+5}{6}, 0\right), \sigma\left(\frac{x+5}{6}, \frac{y+5}{6}\right)\right\} \\
& =\min \left\{\frac{x+5}{6}, \frac{|x-y|}{6}\right\}=\frac{|x-y|}{6} .
\end{aligned}
$$

Therefore, $\quad \delta_{\sigma}(T x, T y)=\frac{|x-y|}{6}$. Similarly, we find $\delta_{\sigma}(T y, T x)=\frac{|x-y|}{6}$. Then, for all $x, y \in K$,

$H_{\sigma}(T x, T y)=\frac{|x-y|}{6}$.

Thus, $H_{\sigma}(T x, T y) \leq k \sigma(x, y)+L \sigma(y, T x)$ for all $x, y \in K$ for all $L \geq 0$. Now, consider the case where $L$ is chosen such that $0<L \leq \frac{3}{10}$.

Note that (2.1) is verified for $z=1$. Moreover, the additional condition $(1+L)(k+2 L)<1$ is also satisfied. 
Hence, $T$ is a multivalued almost contraction that satisfies all assumptions of Theorem 2.1, and $T$ has two fixed points; that is, $\operatorname{Fix}(T)=\{0,1\}$.

Example 2.5 Let $X=\mathbb{R}, K=[0,1]$ and $k=\frac{2}{3}$. Define $T: K \rightarrow C B^{\sigma}(X)$ by $T x=\left\{0, \frac{x^{2}+2}{3}\right\}$, for all $x \in K$ and $\sigma(x, y)=|x-y|$. Recall that Rothe's type condition is verified. We show that $T$ is a multivalued almost contraction. In fact, we have for all $x, y \in[0,1]$

$$
\begin{aligned}
H_{\sigma}(T x, T y)= & \max \left\{\delta_{\sigma}(T x, T y), \delta_{\sigma}(T y, T x)\right\} \\
= & \max \left\{\max \left\{\sigma(0, T y), \sigma\left(\frac{x^{2}+2}{3}, T y\right)\right\},\right. \\
& \left.\max \left\{\sigma(0, T x), \sigma\left(\frac{y^{2}+2}{3}, T x\right)\right\}\right\} .
\end{aligned}
$$

It is easy to show that $\sigma(0, T y)=0$. We have

$$
\begin{aligned}
\sigma\left(\frac{x^{2}+2}{3}, T y\right) & =\min \left\{\sigma\left(\frac{x^{2}+2}{3}, b\right): b \in T y\right\} \\
& =\min \left\{\sigma\left(\frac{x^{2}+2}{3}, 0\right), \sigma\left(\frac{x^{2}+2}{3}, \frac{y^{2}+2}{3}\right)\right\} \\
& =\min \left\{\frac{x^{2}+2}{3}, \frac{\left|x^{2}-y^{2}\right|}{3}\right\}=\frac{\left|x^{2}-y^{2}\right|}{3} .
\end{aligned}
$$

Therefore, $\quad \delta_{\sigma}(T x, T y)=\frac{\left|x^{2}-y^{2}\right|}{3}$. Similarly, we find $\delta_{\sigma}(T y, T x)=\frac{\left|x^{2}-y^{2}\right|}{3}$. Then, for all $x, y \in K$,

$H_{\sigma}(T x, T y)=\frac{\left|x^{2}-y^{2}\right|}{3}$.

Thus,

$$
\begin{aligned}
H_{\sigma}(T x, T y) \leq & \frac{(x+y)|x-y|}{3} \leq \frac{2}{3}|x-y| \leq k \sigma(x, y) \\
& +L \sigma(y, T x)
\end{aligned}
$$

for all $x, y \in K$ and for all $L \geq 0$. Now, consider the case where $L$ is chosen such that $0 \leq L \leq \frac{1}{4+\sqrt{22}}$.

Note that (2.1) is verified for $z=0$ if $y \leq 0$ and for $z=1$ if $y \geq 1$. Moreover, the additional condition $(1+L)(k+$ $2 L)<1$ is also satisfied.

Hence, $T$ is a multivalued almost contraction that satisfies all assumptions of Theorem 2.1, and $T$ has two fixed points; that is, $\operatorname{Fix}(T)=\{0,1\}$.

Example 2.6 Let $X=\mathbb{R}, K=[0,1], k=\frac{1}{4}$. Define $T$ : $K \rightarrow C B^{\sigma}(X)$ by $T x=\left\{0, \frac{1}{2+x}\right\}$, for all $x \in K$ and $\sigma(x, y)=|x-y|$. Recall that Rothe's type condition is verified. We show that $T$ is a multivalued almost contraction. In fact, we have for all $x, y \in[0,1]$

$$
\begin{aligned}
H_{\sigma}(T x, T y)= & \max \left\{\delta_{\sigma}(T x, T y), \delta_{\sigma}(T y, T x)\right\} \\
= & \max \left\{\max \left\{\sigma(0, T y), \sigma\left(\frac{1}{2+x}, T y\right)\right\},\right. \\
& \left.\max \left\{\sigma(0, T x), \sigma\left(\frac{1}{2+y}, T x\right)\right\}\right\} .
\end{aligned}
$$

It is easy to show that $\sigma(0, T y)=0$. We have also,

$$
\begin{aligned}
\sigma\left(\frac{1}{2+x}, T y\right) & =\min \left\{\sigma\left(\frac{1}{2+x}, b\right): b \in T y\right\} \\
& =\min \left\{\sigma\left(\frac{1}{2+x}, 0\right), \sigma\left(\frac{1}{2+x}, \frac{1}{2+y}\right)\right\} \\
& =\min \left\{\frac{1}{2+x}, \frac{|x-y|}{(2+x)(2+y)}\right\} \\
& =\frac{|x-y|}{(2+x)(2+y)} .
\end{aligned}
$$

Therefore, $\delta_{\sigma}(T x, T y)=\frac{|x-y|}{(2+x)(2+y)}$. Similarly, we find $\delta_{\sigma}(T y, T x)=\frac{|x-y|}{(2+x)(2+y)}$. Then, for all $x, y \in K$,

$H_{\sigma}(T x, T y)=\frac{|x-y|}{(2+x)(2+y)}$.

Thus,

$H_{\sigma}(T x, T y) \leq \frac{1}{4}|x-y| \leq k \sigma(x, y)+L \sigma(y, T x)$

for all $x, y \in K$ and for all $L \geq 0$. Now, consider the case where $L$ is chosen such that $0 \leq L \leq \frac{1}{4}$.

Note that (2.1) is verified for $z=0$ if $y \leq 0$ and for $z=1$ if $y \geq 1$. Moreover, the additional condition $(1+L)(k+$ $2 L)<1$ is also satisfied.

Then, $T$ is a multivalued almost contraction that satisfies all assumptions of Theorem 2.1, and $T$ has two fixed points; that is, $\operatorname{Fix}(T)=\{0,-1+\sqrt{2}\}$.

Example 2.7 Let $X=\{0,1,2\}, K=\{0,1\} \quad$ and $k \in[0,1)$. Consider $\sigma: X \times X \rightarrow[0, \infty)$ defined by

$$
\begin{aligned}
& \sigma(0,0)=\sigma(1,1)=0, \sigma(2,2)=\frac{1}{4}, \sigma(1,0)=\sigma(0,1)=\frac{1}{3}, \\
& \sigma(2,0)=\sigma(0,2)=\frac{3}{5} \text { and } \sigma(1,2)=\sigma(2,1)=\frac{2}{5} .
\end{aligned}
$$

Then, $(X, \sigma)$ is a complete metric-like space. Define $T$ : $K \rightarrow C B^{\sigma}(X)$ by

$T 0=T 1=\{0,1\}$.

Note that $T x$ is bounded for all $x \in X$ in the metric-like space $(X, \sigma)$ and the Rothe's type condition is verified. 
About the closedness of $T x$ in $(X, \sigma)$, mention that $T x=$ $\{0,1\} \subset \overline{\{0,1\}}=\overline{T x}$ for all $x \in K$. While, if $2 \in \overline{\{0,1\}}$, so there exists $x \in\{0,1\}$, such that

$$
\sigma(2, x)<\sigma(2,2)+\varepsilon=\frac{1}{4}+\varepsilon, \quad \forall \varepsilon>0,
$$

which is a contradiction due to $\sigma(2,0)=\frac{3}{5}$ and $\sigma(2,1)=\frac{2}{5}$. So, $2 \notin \overline{\{0,1\}}$. Similarly, it is clear that $0 \in \overline{\{0,1\}}$ and $1 \in \overline{\{0,1\}}$, then $\overline{\{0,1\}} \subset\{0,1\}$. We conclude that $T x=\overline{T x}$ for all $x \in X$, that is, $T x$ is closed in $(X, \sigma)$. We also have

$$
\begin{aligned}
H_{\sigma}(T x, T y) & =H_{\sigma}(\{0,1\},\{0,1\}) \\
& =\max \{\sigma(0,\{0,1\}), \sigma(1,\{0,1\})\} \\
& =\sigma(1,\{0,1\}) \\
& =\min \{\sigma(0,1), \sigma(1,1)\} \\
& =0 \leq k \sigma(x, y)+L \sigma(y, T x)
\end{aligned}
$$

for all $x, y \in K$ and for all $L \geq 0$. Now, consider the case where $L$ is chosen such that $(1+L)(k+2 L)<1$. Note that (2.1) is verified for $z=0$ if $x=0$ and $y=2$ and for $z=1$ if $x=1$ and $y=2$. Therefore, $T$ is a multivalued almost contraction that satisfies all assumptions of Theorem 2.1, and $T$ has two fixed points; that is, $\operatorname{Fix}(T)=\{0,1\}$.

Acknowledgments The authors are grateful to the referees for their helpful comments leading to improvement of the presentation of the work.

Open Access This article is distributed under the terms of the Creative Commons Attribution 4.0 International License (http:// creativecommons.org/licenses/by/4.0/), which permits unrestricted use, distribution, and reproduction in any medium, provided you give appropriate credit to the original author(s) and the source, provide a link to the Creative Commons license, and indicate if changes were made.

\section{References}

1. Aage, C.T., Salunke, J.N.: Some results of fixed point theorem in dislocated quasi metric space. Bull. Marathadawa Math. Soc. 9, 1-5 (2008)

2. Aage, C.T., Salunke, J.N.: The results of fixed points in dislocated and dislocated quasi metric space. Appl. Math. Sci. 2, 2941-2948 (2008)
3. Alghamdi, M.A., Berinde, V., Shahzad, N.: Fixed points of nonself almost contractions. J. Appl. Math. 2013(621614), 6 (2013)

4. Aydi, H., Felhi, A., Karapinar, E., Sahmim, S.: Hausdorff MetricLike, generalized Nadler's fixed point theorem on metric-like spaces and application. (in press)

5. Assad, N.A., Kirk, W.A.: Fixed point theorems for set-valued mappings of contractive type. Pac. J. Math. 43, 553-562 (1972)

6. Berinde, V.: On the approximation of fixed points of weak contractive mappings. Carpath. J. Math. 19(1), 7-22 (2003)

7. Berinde, V.: Approximating fixed points of weak contractions using the Picard iteration. Nonlinear Anal. Forum. 9(1), 43-53 (2004)

8. Berinde, M., Berinde, V.: On a general class of multi-valued weakly Picard mappings. J. Math. Anal. Appl. 326(2), 772-782 (2007)

9. Ćirić, L.B.: A remark on Rhoades fixed point theorem for nonself mappings. Int. J. Math. Math. Sci. 16(2), 397-400 (1993)

10. Assad, N.A.: A fixed point theorem for some non-selfmappings. Tamkang J. Math. 21(4), 387-393 (1990)

11. Amini, A.: Harandi, Metric-like spaces, partial metric spaces and fixed points. Fixed Point Theory Appl. 2012, 204 (2012)

12. Banach, S.: Sur les opérations dans les ensembles abstraits et leur application aux équations intégrales. Fund. Math. 3, 133-181 (1922)

13. Karapınar, E., Salimi, P.: Dislocated metric space to metric spaces with some fixed point theorems. Fixed Point Theory Appl. 2013, 222 (2013)

14. Khojasteh, F., Rakocevic, V.: Some new common fixed point results for generalized contractive multi-valued non-selfmappings. Appl. Math. Lett. 25(3), 287-293 (2012)

15. Kohli, M., Shrivastava, R., Sharma, M.: Some results on fixed point theorems in dislocated quasi metric space. Int. J. Theoret. Appl. Sci. 2, 27-28 (2010)

16. Nadler, S.B.: multivalued contraction mappings. Pac. J. Math. 30, 475-488 (1969)

17. Phiangsungnoen, S., Kumam, P.: Ulam-Hyers stability results for fixed point problems via generalized multivalued almost contraction. Proceedings of the International MultiConference of Engineers and Computer Scientists 2014, Hong Kong (2014) 12-14 March, pp. 1222-1225

18. Phiangsungnoen, S., Kumam, P.: Fuzzy fixed point theorems for multivalued fuzzy contractions in b-metric spaces. J. Nonlinear Sci. Appl. 8, 55-63 (2015)

19. Phiangsungnoen, S., Wairojjana, N., Kumam, P.: Fixed point theorem and stability for $(\alpha, \psi, \xi)$-generalized contractive multivalued mappings. Trans. Eng. Technol. 127-139 (2015)

20. Zeyada, Z.M., Hassan, G.H., Ahmad, M.A.: A generalization of fixed point theorem due to Hitzler and Seda in dislocated quasi metric space. Arab. J. Sci. Eng. 31, 111-114 (2005)

21. Zoto, K., Houxha, E., Isufati, A.: Some new results in dislocated and dislocated quasi metric space. Appl. Math. Sci. 6, 3519-3526 (2012) 